\title{
The Scotland and Newcastle epidemiological study of Hodgkin's disease: impact of histopathological review and EBV status on incidence estimates
}

\author{
R F Jarrett, A S Krajewski, B Angus, J Freeland, P R Taylor, G M Taylor, F E Alexander
}

J Clin Pathol 2003;56:811-816

See end of article for authors' affiliations

Correspondence to: Professor R F Jarrett, LRF Virus Centre, Institute of Comparative Medicine, Faculty of Veterinary Medicine, University of Glasgow, Glasgow, G61 1QH, UK; r.f.jarrett@ vet.gla.ac.uk

Accepted for publication 11 May 2003
Aims: The epidemiological and pathological features of Hodgkin lymphoma $(\mathrm{HL})$ are complex. The Epstein-Barr virus (EBV) is consistently associated with a proportion of cases, and these cases are thought to represent a distinct aetiological subgroup of $\mathrm{HL}$. The aim of the present analysis was to determine the age and sex specific incidence of EBV associated and non-associated $\mathrm{HL}$, analysed separately, using data derived from a population based study-the Scotland and Newcastle epidemiological study of Hodgkin's disease (SNEHD). This study also provided a unique opportunity to evaluate accuracy in the current diagnosis and classification of $\mathrm{HL}$.

Methods: SNEHD analysed consecutive cases of HL diagnosed in the study area between 1993 and 1997. Diagnostic biopsy material was retrieved, EBV status of tumours was determined, and histological review was performed.

Results: In total, 622 cases were eligible for the study, and EBV studies and histopathological review were performed on biopsy material from 537 and 549 cases, respectively. Accuracy in the overall diagnosis of $\mathrm{HL}$ and classification of nodular sclerosis $\mathrm{HL}$ was good, but diagnosis of $\mathrm{HL}$ in the elderly and classification of other subtypes was less reliable. One third of classic $\mathrm{HL}$ cases were EBV associated, and age specific incidence curves for EBV associated and non-associated cases were distinct.

Conclusions: Comparison of age specific incidence curves for EBV associated and non-associated $\mathrm{HL}$ supports the hypothesis that these are two distinct aetiological entities. Accuracy in the diagnosis of $\mathrm{HL}$ is generally good, but certain subgroups of cases continue to present diagnostic difficulties.
$\mathrm{H}$ odgkin lymphoma (HL) is a heterogeneous condition that most probably comprises more than one aetiological entity. ${ }^{1}$ Recent data suggest that the Epstein-Barr virus (EBV) is associated with approximately one third of cases in developed countries, and this association is believed to be causal..$^{2-4}$ Data describing the association between EBV and HL have mostly been derived from studies examining archival samples and reporting the proportion of positive samples within subgroups of cases. ${ }^{3-5}$ It is clear from these studies that EBV is more often associated with mixed cellularity HL (MCHL) than nodular sclerosis HL (NSHL), and that HL in children and older adults is more likely to be EBV associated than is HL in young adults..$^{5-8}$ The only population based studies that have classified cases according to EBV status have been small or restricted by age or sex. ${ }^{9-12}$ The epidemiology of HL is complex, and further understanding of the aetiology of this heterogeneous condition will only be gained by performing epidemiological studies with separate analyses of EBV associated and non-associated cases, because these are thought to represent two distinct aetiological entities. Therefore, we set up the Scotland and Newcastle epidemiological study of Hodgkin's disease (SNEHD), a population based, case-control study of adult HL (then referred to as Hodgkin's disease). Our main aim was to identify risk factor profiles for the development of HL, with stratification of cases by EBV status of tumours. Because SNEHD is population based and relatively large, it has enabled us, for the first time, to generate separate age and sex specific incidence curves for EBV associated and nonassociated HL.

"The epidemiology of Hodgkin lymphoma is complex, and further understanding of the aetiology of this heteroge- neous condition will only be gained by performing epidemiological studies with separate analyses of Epstein-Barr virus associated and non-associated cases, because these are thought to represent two distinct aetiological entities"

Unlike non-Hodgkin lymphomas (NHL), the subclassification of HL has remained relatively stable since the Rye classification was introduced in $1966 .{ }^{13-17}$ Because it is now recognised that Hodgkin and Reed-Sternberg (HRS) cells, the malignant cells in HL, are derived from lymphocytes the term Hodgkin lymphoma is preferred to Hodgkin's disease. ${ }^{15}{ }^{18}$ The main change in subclassification is that nodular lymphocyte predominant HL (NLPHL) with a nodular growth pattern is now considered a separate disease entity, with a distinct immunophenotype and cellular origin. ${ }^{14-16}{ }^{18}$ It is therefore classified separately from other subtypes, which are collectively referred to as classic HL (CHL). ${ }^{17} \mathrm{CHL}$ includes the subtypes NSHL, MCHL, lymphocyte depleted HL (LDHL), and the additional subtype lymphocyte rich classic (LRCHL).

\footnotetext{
Abbreviations: CHL, classic Hodgkin lymphoma; EBER, Epstein-Barr virus encoded RNA; EBV, Epstein-Barr virus; HL, Hodgkin lymphoma; HRS, Hodgkin and Reed-Sternberg; IHC, immunohistochemistry; IM, infectious mononucleosis; LMP1, latent membrane protein 1; LDHL, lymphocyte depleted Hodgkin lymphoma; LRCHL, lymphocyte rich classic Hodgkin lymphoma; MCHL, mixed cellularity Hodgkin lymphoma; NHL, non-Hodgkin lymphoma; NLPHL, lymphocyte predominant Hodgkin lymphoma; NOS, not otherwise specified; NSHL, nodular sclerosing Hodgkin lymphoma; SNEHD, Scotland and Newcastle epidemiological study of Hodgkin's disease; SNLG, Scotland and Newcastle lymphoma group
} 
Many cases previously diagnosed as LDHL are now included within NSHL, or reclassified as NHLs. ${ }^{17}$ Diagnostic accuracy in the diagnosis and subclassification of $\mathrm{HL}$ has been assessed previously ${ }^{19-32}$; however, the only population based study to deal with this issue in recent years was restricted to female patients. ${ }^{33}$ The inclusion of careful histopathological review in SNEHD presented an opportunity to evaluate accuracy in the diagnosis of HL in current practice.

\section{METHODS \\ Cases}

SNEHD is a case-control study, but only index cases are included in the present report. Eligibility criteria for inclusion in SNEHD were: diagnosis of HL between 1 January 1993 and 31 July 1997; age at diagnosis of 16-74 years; residence in Scotland (excluding Dumfries and Galloway and the Western Isles) or the Northern Region of England; and, where known, birth in the UK. Cases were identified from the Scotland and Newcastle Lymphoma Group (SNLG) Registry, the ongoing system for registration of lymphomas already in place in this geographical area. Annual crosschecks were performed with relevant cancer registries (Scottish Cancer Registry and Northern Region Cancer Registry), and this provided a second source of cases. Ethical permission was obtained from all local research ethics committees in the study area, and the pathology working party of the SNLG approved the histological review.

\section{Samples and histopathological review}

Pathology reports and diagnostic biopsy samples, including sections stained using immunohistochemistry (IHC), were retrieved where possible. Immunohistochemical staining was carried out in 232 cases using a panel of monoclonal antibodies reactive with: CD45; CD20; CD3; CD30; CD15; and epithelial membrane antigen (Dako, Ely, Cambridgeshire, UK). Immunostaining was performed using a Dako Techmate automated staining system (Dako) and reactivity was detected using an $\mathrm{ABC}$ technique, according to the manufacturer's instructions (Vector Laboratories, Peterborough, UK). The procedure for histopathological review was designed so that the reviewing pathologists, BA and ASK, would not review cases in which they had made the initial diagnosis and classification. Review diagnoses were returned to the LRF virus centre and, where the review and referral diagnosis differed, or where the diagnosis was considered problematic, cases were referred for discussion and review (BA, ASK, and RFJ) using a multiheaded microscope. Reviewers were blind to the EBV status of the lesions. Cases were initially categorised according to the revised European-American lymphoma classification system, ${ }^{14}$ but the more recent World Health Organisation nomenclature is reported here. ${ }^{34}$

\section{EBV status}

The EBV status of tumours was determined using EBV encoded RNA (EBER) in situ hybridisation. The EBER assay was carried out using a commercially available oligonucleotide probe (Vector Laboratories) and hybridisation detection kit (Dako). In the few cases in which results were difficult to interpret, or in which there appeared to be large numbers of EBV positive cells in the reactive component of lesions, latent membrane protein l (LMPl) IHC was performed. After antigen retrieval, the CSI-4 cocktail of monoclonal antibodies (Dako) was applied to paraffin wax embedded sections and reactivity was detected using an $\mathrm{ABC}$ kit (Vector Laboratories) incorporating diaminobenzidine (Dako) as the chromogenic substrate. Cases were considered EBV associated or EBV+ HL if HRS cells were positive in either assay.

\section{Statistical methods}

The $\kappa$ coefficient was used to measure the level of agreement, corrected for chance, between referral and review diagnoses in six diagnostic categories: NLPHL, MCHL, LDHL, NSHL, LRCHL, and CHL not otherwise specified (NOS). The age and sex specific incidence rates were computed for age groups 16$24,25-34,35-49,50-59,60-69$, and older people using as denominators population counts from the 1991 census. Comparisons of proportions have been tested using Pearson's $\chi^{2}$ test. All statistical analyses were implemented using SPSS.

\section{RESULTS \\ Patients}

A total of 622 individuals were identified who fulfilled eligibility criteria and had a confirmed diagnosis of HL. Biopsy material was retrieved from 561 patients and in 549 was considered suitable for review. Appropriate material for histopathological review was not available in 73 cases; these cases have been retained in some analyses.

\section{Histopathological review}

Haematoxylin and eosin stained sections from 549 cases were subjected to histopathological review. IHC staining was performed in 232 cases and in an additional 113 cases the original IHC was available; therefore, IHC was reviewed in 345 cases. In a further 117 cases, IHC results were obtained from the original pathology report. In the remaining 87 cases, IHC could not be assessed; however, in 72 of these IHC was considered unnecessary for diagnostic purposes. Histological subtyping of all tumours was not possible, but attempts were made to distinguish NLPHL from CHL, and the diagnostic category CHL NOS was therefore introduced.

HL was confirmed in 511 of the 549 cases (93\%) subjected to expert review, including all cases in which the IHC could not be assessed (table 1). In 26 cases $(4.7 \%)$, the diagnosis of HL was revised; in 23 instances (4.1\%), the diagnosis was changed to NHL, and in three cases only reactive changes were observed in the material submitted for review. Among cases reclassified as NHL, diffuse large B cell lymphoma $(\mathrm{n}=9)$ and $\mathrm{T}$ cell rich $\mathrm{B}$ cell lymphoma $(\mathrm{n}=9)$ were the most common review diagnoses. Cases with a referral diagnosis of NLPHL $(n=5)$, MCHL $(n=7)$, and HL NOS ( $\mathrm{n}=7$ ) were over-represented among cases with a revised diagnosis, as were patients in the older age group (see below). For the 33 cases with an initial diagnosis of HL NOS or CHL NOS, HL was confirmed in only 21 cases $(63.6 \%)$. In 12 of the 549 cases $(2 \%)$, the diagnosis of HL was considered uncertain following pathological review, either because the biopsy material was inadequate $(n=10)$ or because a firm diagnosis could not be reached without further investigation $(\mathrm{n}=2)$. These cases have been classified as "not HL" in this and all further analyses of SNEHD.

The histological subtype at initial diagnosis was known for 534 cases; the referral and review diagnoses were identical in 386 instances, giving $72.3 \%$ agreement overall and $75.6 \%$ for cases confirmed as HL. There were 23 changes $(4.3 \%)$ between NLPHL and CHL, now considered distinct disease entities. Additional subtype changes occurred in 86 instances, with MCHL to NSHL being the most frequent diagnostic change $(n=34)$. Confirmation rates (the percentage of referral diagnoses confirmed on review) differed for the different histological subtypes, being good for NSHL (88\%), but less good for NLPHL (64\%) and MCHL (59\%). For female patients the confirmation rate for NSHL was slightly higher (92\%), but dropped to $46 \%$ and $43 \%$ for NLPHL and MCHL, respectively. There were 12 cases with a referral diagnosis of LDHL, but only one of these was confirmed on review, giving a confirmation rate of $8.3 \%$; only five cases in the total series 
Table 1 Comparison between referral and review diagnoses

\begin{tabular}{|c|c|c|c|c|c|c|c|c|c|c|}
\hline \multirow[b]{2}{*}{ Referral diagnosis } & \multicolumn{10}{|c|}{ Review diagnosis } \\
\hline & NLPHL & MCHL & LDHL & NSHL & LRCHL & CHL NOS & NHL & Reactive & $\begin{array}{l}\text { Diagnosis } \\
\text { uncertain }\end{array}$ & $\begin{array}{l}\text { Confirmation } \\
\text { rate }\end{array}$ \\
\hline NLPHL & 36 & 3 & 0 & 8 & 3 & 0 & 5 & 0 & 1 & $64.3 \%$ \\
\hline $\mathrm{MCHL}$ & 2 & 79 & 0 & 34 & 7 & 5 & 7 & 0 & 1 & $58.5 \%$ \\
\hline LDHL & 0 & 4 & 1 & 3 & 0 & 2 & 0 & 0 & 2 & $8.3 \%$ \\
\hline NSHL & 5 & 14 & 0 & 258 & 1 & 4 & 4 & 0 & 6 & $88.3 \%$ \\
\hline LRCHL & 0 & 0 & 0 & 1 & 5 & 0 & 0 & 0 & 0 & $83.3 \%$ \\
\hline CHL NOS & 0 & 1 & 0 & 11 & 0 & 7 & 0 & 0 & 2 & \\
\hline HL NOS & 0 & 0 & 0 & 0 & 0 & 2 & 7 & 3 & 0 & \\
\hline HL subtype not known & 2 & 2 & 1 & 8 & 0 & 2 & 0 & 0 & 0 & \\
\hline Identification rate & $80 \%$ & $76.7 \%$ & $50 \%$ & $79.9 \%$ & $31.2 \%$ & & & & & \\
\hline
\end{tabular}

Confirmation rates indicate the percentage of cases with referral diagnosis confirmed on review; identification rates indicate the percentage of review diagnoses identified at referral.

CHL NOS, classic Hodgkin lymphoma not otherwise specified; HL NOS, Hodgkin lymphoma not otherwise specified; LDHL, lymphocyte depleted Hodgkin lymphoma; LRCHL, lymphocyte rich classic Hodgkin lymphoma; MCHL, mixed cellularity Hodgkin lymphoma; NLPHL, nodular lymphocyte predominant Hodgkin lymphoma; NSHL, nodular sclerosing Hodgkin lymphoma.

and two of the reviewed cases were classified as LDHL, confirming that this subtype is almost disappearing with the use of modern diagnostic criteria. ${ }^{17}$ Identification rates (the percentage of review diagnoses that were the same as initial diagnosis) showed less variation among the major subtypes (HLNS, 80\%; NLPHL, 80\%; MCHL, 77\%); this value was much lower for LRCHL $(31 \%)$, but this reflects the introduction of this subtype as a provisional entity during the study period. ${ }^{14}$ The $\kappa$ coefficient for chance corrected agreement between referral and review diagnosis using six categories (NLPHL, MCHL, LDHL, NSHL, LRCHL, and CHL NOS) is 0.62, confirming a substantial level of agreement.

Diagnostic changes were analysed with respect to patient age in three age groups: 16-34 years, 35-49 years, and $>50$ years. Changes in diagnosis and subtype were more common for the older adult age group, and differences were statistically significant for "all changes" $(p<0.001)$, and changes from HL to "diagnosis uncertain". There was also a higher proportion of cases in the older adult group in which histological subtyping could not be reliably performed $(\mathrm{p}=0.01)$.

Data from the 511 confirmed cases of HL, plus the 73 cases not subjected to histopathological review, were used to derive the incidence of HL by age, sex, and histological subtype (fig 1). A bimodal curve with an incidence peak in young adulthood (15-34 years) and a second peak in older adults ( $\geqslant 55$ years) is evident for "all HL" and CHL. Overall, 61.3\% of cases were classified as NSHL and $21.4 \%$ as MCHL. NSHL largely accounts for the young adult age incidence peak, whereas the incidence of MCHL shows less variation by age (fig 1B). Age incidence patterns for the sexes are different; there is a male excess up to 65 years, with the curve for women showing more evidence of bimodality (fig lC).

\section{EBV status}

EBV status was determined for 537 cases, including 461 cases with a review diagnosis of CHL; in 154 (33\%) of these last cases all of the HRS cells were EBER positive and therefore designated EBV associated or EBV+ HL (fig 2; table 2). LMPI staining identified no EBV+ HL cases that were not picked up using the EBER assay. Consistent with previous studies, there were significant differences by histological subtype, with 63 of $105(60 \%)$ MCHL and 76 of 320 (23.8\%) NSHL cases being EBV associated, respectively $(\mathrm{p}<0.001)$. Differences by age group were also highly significant, with patients who have CHL in the older adult group (71 of $137 ; 51.8 \%$ ) being more likely to have EBV+ HL than young adult patients (54 of 236; $22.8 \%)(p<0.001)$. Table 2 shows the number of patients with EBV+ HL in each five year age group. There was a male excess within EBV+ HL (male to female ratio, 102:52) but not EBV - HL (male to female ratio, 152:155) ( $<<0.001)$.

Age and sex specific incidence curves for EBV associated and non-associated HL in adults were determined and, as
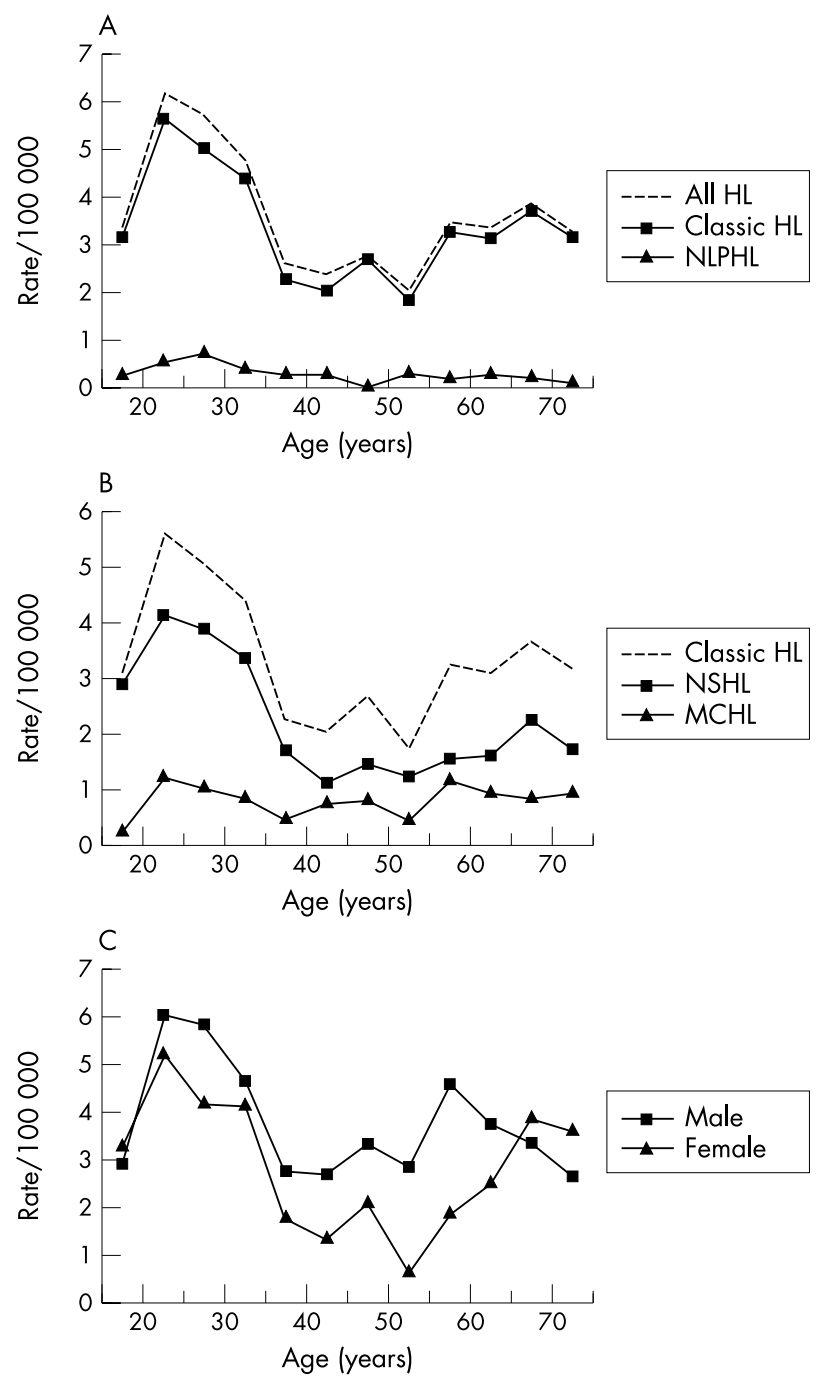

Figure 1 Age specific incidence rates of Hodgkin lymphoma $(\mathrm{HL})$ for each 100000 person years. (A) All HL, classic HL, and nodular lymphocyte predominant $\mathrm{HL}$ (NLPHL). (B) Classic $\mathrm{HL}$, nodular sclerosis $\mathrm{HL}$ (NSHL), and mixed cellularity HL (MCHL). (C) Classic HL by sex. 

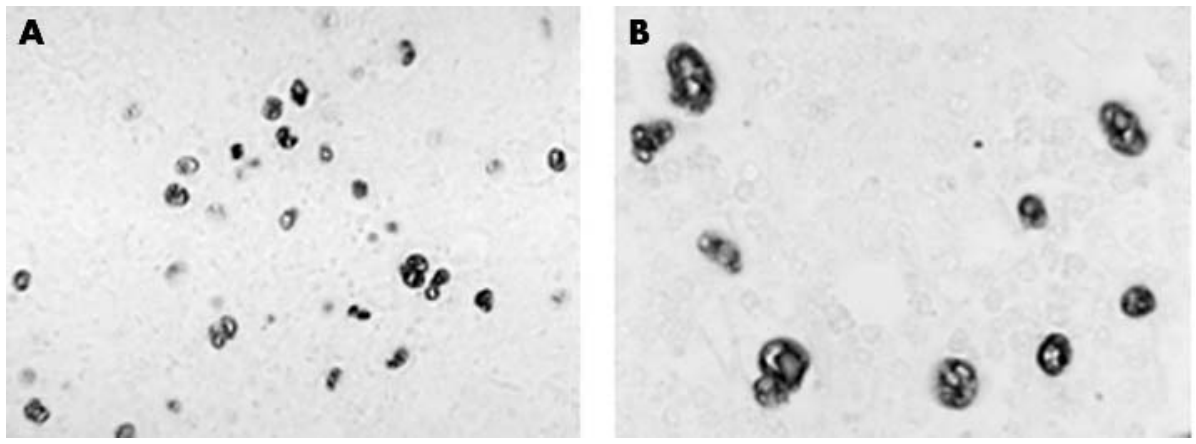

Figure 2 Representative Epstein-Barr virus encoded RNA in situ hybridisations showing the characteristic staining pattern in the nuclei of Hodgkin and Reed-Sternberg cells. (A) Low power and (B) high power view.

predicted, these have different shapes (fig 3). The non-EBV associated cases show the young adult peak, and the incidence then declines with age. In contrast, the curve for EBV+ HL has a peak in the older adult age group; a smaller peak is also observed at the start of the young adult age range, consistent with our previous prediction. ${ }^{11}$ Bimodality of the incidence curve for EBV+ HL persists when male and female patients are analysed separately (fig 3B).

\section{DISCUSSION}

SNEHD provided a unique opportunity to investigate the age incidence of HL, stratified by EBV status, and the impact of expert histological review on HL diagnosis and classification. It is the largest population based study of HL to examine the EBV status of lesions; the only similar survey is a population based analysis of 395 women aged 19-79 in northern California. ${ }^{33}$ Although the effects of histological review on the diagnosis and classification of HL have been well documented, ${ }^{19-25}$ 27-32 $\mathrm{few}$ analyses have been performed on population based data sets, ${ }^{22}{ }^{24}$ and most were performed before the introduction of modern diagnostic criteria and immunohistochemical staining techniques. ${ }^{19-21} 232527-30$

"Only two of the reviewed cases were considered to be lymphocyte depleted Hodgkin lymphoma (LDHL), consistent with the recognition that many cases previously classified as LDHL are either nodular sclerosing Hodgkin lymphoma or non-Hodgkin lymphoma"

In SNEHD, histological review confirmed the diagnosis of HL in most cases $(93.1 \%)$; only $4.7 \%$ of cases were considered to have diagnoses other than HL, a similar figure to the 3\% reported by Glaser et al..$^{33}$ A further $2.2 \%$ of SNEHD diagnoses

Table 2 Epstein-Barr virus (EBV) association in classic Hodgkin lymphoma by age group

\begin{tabular}{llll}
\hline Age group (years) & All cases & EBV + & EBV - \\
\hline $16-19$ & 40 & 8 & 32 \\
$20-24$ & 69 & 20 & 49 \\
$25-29$ & 64 & 14 & 50 \\
$30-34$ & 63 & 12 & 51 \\
$35-39$ & 29 & 9 & 20 \\
$40-44$ & 28 & 8 & 20 \\
$45-49$ & 32 & 12 & 20 \\
$50-54$ & 21 & 6 & 15 \\
$55-59$ & 31 & 19 & 12 \\
$60-64$ & 29 & 18 & 11 \\
$65-69$ & 33 & 16 & 17 \\
$70-74$ & 22 & 12 & 10 \\
\hline
\end{tabular}

All cases, cases of classic Hodgkin lymphoma with EBV status known; $\mathrm{EBV}+$, number of EBV associated cases; $E B V-$, number of non-EBV associated cases. Differences in the proportion of EBV associated cases by age group are highly significant $(p<0.001)$. were considered uncertain following histological review, and assessment of further biopsy material was deemed necessary before a correct diagnosis could be reached. Overall, this represents a considerable improvement over many previous comparisons. ${ }^{19} 2022272935$ There was more disagreement concerning the histological subtyping of lesions, with $24 \%$ of confirmed HL cases having a different referral and review diagnosis. Consistent with many previous studies, the confirmation rate for a referral diagnosis of NSHL was good, but for MCHL and NLPHL reliability was much lower. ${ }^{21}{ }^{23-33}$ Only two of the reviewed cases were considered to be LDHL, consistent with the recognition that many cases previously classified as LDHL are either NSHL or NHL. ${ }^{17}$ Diagnostic reliability was lower for older patients ( $\geqslant 50$ years), with more misdiagnoses, subtype changes, and uncertainties in this age group. This may, in part, reflect the increased incidence of NHL in older patients; however, it was noted that the quality of biopsy material from older patients was often poor. Therefore, despite improvements in the diagnosis of HL over time, studies evaluating diagnostic accuracy since the implementation of the Rye classification consistently report underdiagnosis of NSHL (although this subtype has
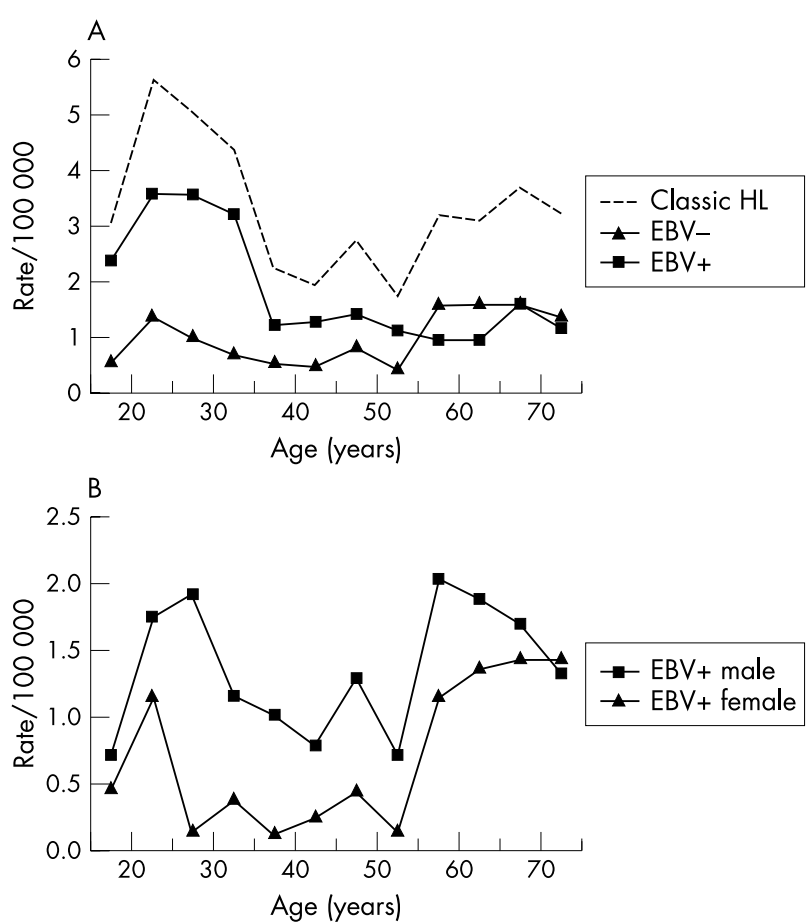

Figure 3 Age specific incidence rates of Hodgkin lymphoma for each 100000 person years. (A) Classic Hodgkin lymphoma (CHL), EBV+ CHL and $E B V-C H L$. (B) EBV+ CHL by sex. 


\section{Take home messages}

- Comparison of the age specific incidence curves for Epstein-Barr virus associated and non-associated Hodgkin lymphoma (HL) supports the hypothesis that these form two distinct aetiological entities

- Although accuracy in the diagnosis of $\mathrm{HL}$ is generally good, certain subgroups of cases and the elderly continue to present diagnostic difficulties

good confirmation rates), and less reliable diagnosis of nonNSHL subtypes and HL in older persons. ${ }^{19-33} 3637$

The data suggest that diagnostic inaccuracy is unlikely to affect overall incidence data for HL although, for the reasons described above, care should be taken in the interpretation of data for subtypes other than NSHL and for persons in the older adult age group ( $\geqslant 50$ years). At the level of the individual patient, there is clearly room for improvement in HL diagnosis. Misdiagnosis of NHL and reactive nodes as HL, and misclassifications between NLPHL and CHL, occurred in $\sim 9 \%$ of cases in our study, and would probably affect clinical management. It is recognised that the differentiation of NLPHL from CHL and NHL is difficult in some cases; however, although the neoplastic cells in NLPHL may show a broad spectrum of morphologies they usually show a characteristic phenotype, with expression of B cell specific markers and lack of expression of CD30 and CD15. ${ }^{38}$ In almost all cases previously classified as diffuse NLPHL, the tumour cells are CD30+ and CD15+ and show the clinical behaviour of CHL, and are properly classified as LRCHL. ${ }^{38} 39$ Furthermore, the existence of LRCHL with a nodular growth pattern is now accepted. ${ }^{17} 3840$ Although improved diagnostic criteria should lead to the more accurate diagnosis of NLPHL, the results of our study suggest that expert review is advisable before embarking on less aggressive treatment regimens for this entity.

Incidence data, including subtype distributions, have been derived from this high quality data set. Overall, a bimodal incidence is seen, with the peak incidence in the young adult age group (fig 1). The distribution of histological subtypes in SNEHD is similar to that seen in other large, histologically reviewed data sets from Denmark and Germany, ${ }^{30} 37$ and for women resembles the distribution in northern California. ${ }^{33}$ Male patients predominate overall, but incidence curves for men and women show different features.

SNEHD is population based and relatively large; therefore, for the first time it has been possible to determine the age and sex adjusted incidence of EBV associated and nonassociated HL. Incidence curves for the subgroups are different, supporting the hypothesis that these are distinct aetiological subgroups of HL. The young adult age incidence peak is largely driven by the non-EBV associated cases. A "hit and run" mechanism involving EBV has been suggested in non-EBV associated cases, but evidence for this is currently lacking; ${ }^{41}$ in particular, we have shown that young adult patients with EBV- HL are more likely to be EBV seronegative than are age matched controls. ${ }^{41}$

"For the first time it has been possible to determine the age and sex adjusted incidence of Epstein-Barr virus associated and non-associated Hodgkin lymphoma"

The age incidence curve for the EBV associated cases is flatter; there is a suggestion that this curve is bimodal, with a first peak in the 15-24 year age range and a second peak at ages $>55$ years, although this is difficult to test formally. We previously suggested that there is a small peak in the incidence curve for EBV associated HL in the 15-24 year age range, ${ }^{11}$ and our present data provide support for this prediction. In SNEHD, young adult patients with EBV associated HL were more likely to report previous infectious mononucleosis (IM) and/or IM in a first degree relative than were patients in other subgroups, suggesting that delayed exposure to EBV is a specific risk factor for the development of young adult EBV+ HL. ${ }^{42}$ The number of EBV+ HL cases in the 15-24 year age incidence peak was small and precluded statistical analysis; however, $44 \%$ of these patients reported IM, or IM in a first degree family member, compared with only $17 \%$ of patients with EBV- HL and $9 \%$ of controls.

Our present study is restricted to adult cases; however, there is a body of data showing that HL in early childhood is usually EBV associated. ${ }^{5}$ Taken together, these data suggest that EBV associated HL is more likely to occur at three time periods in life-in early childhood, young adulthood, and older adult life. We predict that EBV associated HL in early childhood and young adulthood is associated with primary EBV infection. Overall, the data support a four disease model of HL, with three groups of EBV associated HL and a group of non-EBV associated cases. ${ }^{43}$

\section{ACKNOWLEDGEMENTS}

We would like to thank all pathologists within the SNLG who contributed material to this study. We particularly thank J White for help with tracing cases and F Jack for discussion of EBV results. SNEHD was funded by the Kay Kendall Leukaemia Fund and work at the LRF virus centre is supported by a Leukaemia Research Fund specialist programme grant.

\section{Authors' affiliations}

R F Jarrett, J Freeland, LRF Virus Centre, Institute of Comparative Medicine, University of Glasgow, Glasgow G61 1QH, UK

A S Krajewski, Department of Pathology, Northampton General NHS Trust, Northampton NN1 5BD, UK

B Angus, Department of Pathology, University of Newcastle upon Tyne, Newcastle upon Tyne NEI 4LP, UK

P R Taylor, Department of Haematology, University of Newcastle upon Tyne

G M Taylor, Immunogenetics Laboratory, University of Manchester, St Mary's Hospital, Manchester M13 OJH, UK

F E Alexander, Department of Community Health, Public Health Sciences, University of Edinburgh, Medical School, Teviot Place, Edinburgh EH8 9AG, UK

\section{REFERENCES}

1 MacMahon B. Epidemiology of Hodgkin's disease. Cancer Res 1966;26:1 189-201.

2 IARC. Proceedings of the IARC working group on the evaluation of carcinogenic risks to humans. Epstein-Barr virus and Kaposi's sarcoma herpesvirus/human herpesvirus 8. Lyon, France, 17-24 June 1997. IARC Monogr Eval Carcinog Risks Hum 1997;70:1-492.

3 Glaser SL, Jarrett RF. The epidemiology of Hodgkin's disease. In: Diehl V, ed. Hodgkin's disease. London: Bailliere Tindall, 1996:401-16.

4 Jarrett RF. Epstein-Barr virus and Hodgkin's disease. Epstein Barr Virus Report 1998;5:77-85.

5 Jarrett RF, Armstrong AA, Alexander E. Epidemiology of EBV and Hodgkin's lymphoma. Ann Oncol 1996;7:S5-S10.

6 Pallesen G, Hamilton-Dutoit SJ, Rowe M, et al. Expression of Epstein-Barr virus latent gene products in tumour cells of Hodgkin's disease. Lancet $1991 ; 337: 320-2$.

7 Armstrong AA, Alexander FE, Paes RP, et al. Association of Epstein-Barr virus with pediatric Hodgkin's disease. Am J Pathol 1993;142:1683-8.

8 Armstrong AA, Alexander FE, Cartwright R, et al. Epstein-Barr virus and Hodgkin's disease: further evidence for the three disease hypothesis. Leukemia 1998; 12:1272-6.

9 Poppema S, Visser L. Epstein-Barr virus positivity in Hodgkin's disease does not correlate with an HLA A2-negative phenotype. Cancer 1994;73:3059-63.

10 Alexander FE, Jarrett RF, Lawrence D, et al. Risk factors for Hodgkin's disease by Epstein-Barr virus (EBV) status: prior infection by EBV and other agents. Br J Cancer 2000;82:1117-21. 
11 Alexander FE, Jarrett RF, Cartwright RA et al. Epstein-Barr Virus and HLADPB 1-*0301 in young adult Hodgkin's disease: evidence for inherited susceptibility to Epstein-Barr virus in cases that are EBV(+ve). Cancer Epidemiol Biomarkers Prev 2001;10:705-9.

12 Clarke CA Glaser SL, Dorfman RF, et al. Epstein-Barr virus and survival after Hodgkin disease in a population-based series of women. Cancer 2001;91:1579-87.

13 Lukes RJ, Butler JJ. The pathology and nomenclature of Hodgkin's disease. Cancer Res 1966;26:1063-83.

14 Harris NL, Jaffe ES, Stein H, et al. A revised European-American classification of lymphoid neoplasms: a proposal from the international lymphoma study group. Blood 1994;84:1361-92.

15 Stein H. Hodgkin lymphomas: introduction. In: Jaffe ES, Harris NL, Stein H, et al, eds. World Health Organisation classification of tumours. Pathology and genetics of tumours of haematopoietic and lymphoid tissues. Lyon: IARC Press, 2001:237-9.

16 Stein H, Delsol G, Pileri S, et al. Nodular lymphocyte predominant Hodgkin lymphoma. In: Jaffe ES, Harris NL, Stein $\mathrm{H}$, et al, eds. World Health organisation classification of tumours. Pathology and genetics of tumours of haematopoietic and lymphoid tissues. Lyon: IARC Press, 2001:240-43.

17 Stein H, Delsol G, Pileri S, et al. Classical Hodgkin lymphoma. In: Jaffe ES, Harris NL, Stein H, et al, eds. World Health Organisation classification of tumours. Pathology and genetics of tumours of haematopoietic and lymphoid tissues. Lyon: IARC Press, 2001:244-253.

18 Kuppers R, Rajewsky K. The origin of Hodgkin and Reed/Sternberg cells in Hodgkin's disease. Annu Rev Immunol 1998;16:471-93.

19 Coppleson LW, Factor RM, Strum SB, et al. Observer disagreement in the classification and histology of Hodgkin's disease. J Natl Cancer Inst 1970:45:731-40

20 Correa P, O'Conor GT, Berard CW, et al. International comparability and reproducibility in histologic subclassification of Hodgkin's disease. J Natl Cancer Inst 1973:50:1429-35.

21 Jones SE, Butler JJ, Byrne GE Jr, et al. Histopathologic review of lymphoma cases from the southwest oncology group. Cancer 1977;39:1071-6.

22 Franssila KO, Heiskala MK, Heiskala HJ. Epidemiology and histopathology of Hodgkin's disease in Finland. Cancer 1977;39:1280-8.

23 Woodruff RD. Reviewing histologic diagnosis of lymphoma: comparison of original and review diagnoses in 269 cases. Arch Pathol Lab Med $1981 ; 105: 573-6$

24 Matz LR, Finlay-Jones LR, Waters ED, et al. The Rye classification of a population based series of Hodgkin's disease patients in Western Australia. Pathology 1981;13:267-76.

25 Miller TP, Byrne GE, Jones SE. Mistaken clinical and pathologic diagnoses of Hodgkin's disease: a southwest oncology group study. Cancer Treat Rep 1982;66:645-51

26 Velez-Garcia E, Durant J, Gams R, et al. Results of a uniform histopathologic review system of lymphoma cases. A ten-year study from the southeastern cancer study group. Cancer 1983;52:675-9.

27 Kim H, Zelman RJ, Fox MA, et al. Pathology panel for lymphoma clinical studies: a comprehensive analysis of cases accumulated since its inception. J Natl Cancer Inst 1982;68:43-67.
28 Holman CD, Matz LR, Finlay-Jones IR, et al Inter-observer variation in the histopathological reporting of Hodgkin's disease: an analysis of diagnostic subcomponents using kappa statistics. Histopathology 1983;7:399-407.

29 Dige U, Johansson H, Lenner $\mathrm{P}$, et al. Hodgkin's disease in northern Sweden 1971-1981. I. A histopathological reevaluation of 223 cases. Acta Oncol 1991;30:593-6.

30 Lauritzen AF, Specht LK, Nissen NI, et al. Changes over the course of time in histological subclassification of Hodgkin's disease in Denmark. Dan Med Bull 1991;38:84-7.

31 Georgii A, Fischer R, Hubner K, et al. Classification of Hodgkin's disease biopsies by a panel of four histopathologists. Report of 1,140 patients from the German National Trial. Leuk Lymphoma 1993:9:365-70.

32 Glaser SL, Swartz WG. Time trends in Hodgkin's disease incidence. The role of diagnostic accuracy. Cancer 1990;66:2196-204.

33 Glaser SL, Dorfman RF, Clarke CA. Expert review of the diagnosis and histologic classification of Hodgkin disease in a population-based cancer registry: interobserver reliability and impact on incidence and survival rates. Cancer $2001 ; 92: 218-24$

34 WHO. World Health Organisation classification of tumours: pathology and genetics of tumours of hematopoietic and lymphoid tissues. Lyon: IARC, 2001.

35 Jack AS, Cunningham D, Soukop M, et al. Use of Leu M1 and antiepithelial membrane antigen monoclonal antibodies for diagnosing Hodgkin's disease. J Clin Pathol 1986;39:267-70.

36 Bennett MH, MacLennan KA, Vaughan Hudson B, et al. The clinical and prognostic relevance of histopathologic classification in Hodgkin's disease. Prog Surg Pathol 1991:127-51.

37 Bernhards J, Fischer R, Hubner K, et al. Histopathological classification of Hodgkin's lymphomas. Results from the reference pathology of the German Hodgkin trial. Ann Oncol 1992;3(suppl 4):31-3

38 Anagnostopoulos I, Hansmann ML, Franssila K, et al. European task force on lymphoma project on lymphocyte predominance Hodgkin disease: histologic and immunohistologic analysis of submitted cases reveals 2 types of Hodgkin disease with a nodular growth pattern and abundant lymphocytes. Blood 2000;96:1889-99

39 Diehl V, Sextro M, Franklin J, et al. Clinical presentation, course, and prognostic factors in lymphocyte-predominant Hodgkin's disease and ymphocyte-rich classical Hodgkin's disease: report from the European task force on lymphoma project on lymphocyte-predominant Hodgkin's disease. J Clin Oncol 1999;17:776-83.

40 Ashton-Key M, Thorpe PA, Allen JP, et al. Follicular Hodgkin's disease. Am J Surg Pathol 1995; 19:1294-9.

41 Gallagher A, Perry J, Freeland J, et al. Hodgkin lymphoma and Epstein-Barr virus (EBV): no evidence to support hit-and-run mechanism in cases classified as non-EBV-associated. Int J Cancer 2003;104:624-30.

42 Alexander FE, Lawrence DJ, Freeland J, et al. An epidemiological study of index and family infectious mononucleosis and adult Hodgkin's disease (HD): evidence for a specific association with $E B V+v e H D$ in young adults. Int $J$ Cancer 2003; 107:298-302

43 Jarrett RF. Viruses and Hodgkin's lymphoma. Ann Oncol 2002;13(S1):23-9. 
A previous resection from the same site showed a schwannoma with no evidence of malignancy, and similar benign areas are present in the current biopsy. This supports our assumption that this malignant tumour has arisen by transformation from the previous lesion. An issue with this case is the history of previous radiation. It has been reported that irradiation may induce neurofibrosarcoma. ${ }^{5}$ These cases report malignancy arising within previously normal nerves and do not describe the induction of malignancy within a previously benign tumour. Regardless of this possible aetiology, we believe that this case represents malignant transformation within a previously benign vestibular schwannoma, and therefore presents a rare case.

J S Wilkinson, H Reid, G R Armstrong Department of Histopathology, Hope Hospital, Stot Lane, Salford, M6 8HD, UK; helen.reid@srht.nhs.uk

\section{References}

1 Mc Lean CA, Laidlaw JD, Brownhill DSB, et al Recurrence of acoustic neurilemmoma as a malignant spindle cell neoplasm. J Neurosurg 1990;73:946-50.

2 Yousem SA, Colby TV, Urich H. Malignant epithelioid schwannoma arising in a benign schwannoma. Cancer 1985:55:2799-803.

3 Kudo M, Matsumoto M, Terao H. Malignant nerve sheath tumour of acoustic nerve. Arch Pathol Lab Med 1983; 107:293-7

4 Carstens PH, Schrodt GR. Malignant transformation of a benign encapsulated neurilemmoma. Am J Clin Pathol 1969;51:144-9.

5 Ducatman BS, Scheithauer BW. Postirradiation neurofibrosarcoma. Cancer 1983;51:1028-33.

\section{Best practice guideline on microbiological investigation of infertility requires further review}

The best practice guideline on the investigation of infertility briefly comments on appropriate microbiological investigations. ${ }^{1}$ However, there are several issues that we feel merit further consideration.

The need to check the rubella immunity status of the female partner is highlighted This point is also stressed by the Royal College of Obstetricians and Gynaecologists (RCOG) ${ }^{2}$ Testing for blood borne viruses (antibodies to hepatitis B surface antigen, human immunodeficiency virus, and hepatitis C) is also commented upon in the bes practice guideline as a general investigation and has been similarly suggested in a recent clinical review. ${ }^{3}$ However, no such guideline has been issued by either the RCOG or the British Fertility Society. Nevertheless, the Human Fertilisation and Embryology Authority has set a deadline of the end of 2004 for the screening of all women/couples participating in licensed infertility treatments (in vitro fertilisation, intracytoplasmic sperm injection, donor gamete therapy) for blood borne viruses.

The wisdom of this approach is questionable for two reasons. First, if testing of subfertile couples is part of the continuum of their care from preconception to birth then why repeat the process when pregnant women will routinely be offered blood borne virus (and syphilis) screening during their antenatal care? Second, because the prevalence of blood borne virus infection in patients seeking infertility advice will probably be low (possibly $<1 \%$ ), where is the evidence that universal blood borne virus screening is cost effective?

We believe that scarce financial resources would be better spent on a screening programme for asymptomatic chlamydia infection in the infertile population. This should be based on a chlamydia molecular amplification test, using urine, lower vaginal swabs, or endocervical swabs, and not chlamydia serology, as has been suggested previously. ${ }^{3}$ Screening for chlamydia is not mentioned in the best practice guideline but is recommended by the RCOG. This is particularly important in women who will be undergoing uterine insemination as part of their fertility investigation or treatment. In general, this will mean routinely testing women less than 25 years of age. ${ }^{4}$ One in 10 sexually active women in England is currently thought to be infected with chlamydia. ${ }^{5}$ Those identified as chlamydia positive could then be offered blood borne virus screening linked to a genitourinary counselling service.

Finally, the best practice guideline makes no comment on screening for cytomegalovirus immunity. Although not routinely recommended, cytomegalovirus IgG testing should be considered both for women who receive donor gametes (sperm or oocytes) and the donors of such gametes.

R P D Cooke, D K C Chui

East Sussex Hospitals NHS Trust, Department of Medical Microbiology, Kings Drive, Eastbourne, East Sussex BN21 2UD, UK Richard.Cooke@aht.nwest.nhs.uk

\section{References}

1 Williams C, Giannopoulos T, Sherriff EA. Investigation of infertility with the emphasis on laboratory testing and with reference to radiological imaging. J Clin Pathol 2003:56:261-7

2 Royal College of Obstetricians and Gynaecologists. The initial investigation and management of the infertile couple. Evidencebased clinical guidelines, No 2. London: RCOG Press, 1998.

3 Cahill DJ, Wardle PG. Management of infertility. BMJ 2002;325:28-32.

4 Royal College of Obstetricians and Gynaecologists. The management of infertility in tertiary care. Evidence-based clinical guidelines, No 6. London: RCOG Press, 2000

5 House of Commons. Sexual health: the third report of session 2002-3. London: HMSO, 2003

\section{BOOK REVIEWS}

\section{Pathology of the Pancreas, Gallbladder, Extrahepatic Biliary Tract and Ampullary Region}

Lack E. (£145.00.) Oxford University Press, 2003. ISBN 0195133927

Superb! The author has succeeded in producing an excellent textbook covering an extensive range of pathology, including both common and exotic entities.

As the title suggests, this book is divided into three parts: "I. Pancreas", "II. Gallbladder", and "III. Extrahepatic biliary tract and ampullary region". The first chapter, entitled "Embryology, anatomy, and function of the exocrine and endocrine pancreas", includes original artwork and makes for very interesting and informative reading.
The approach is extremely methodical and the text is comprehensive. The text is written under appropriate headings/subheadings, which makes it easy to read. Where relevant, there is additional information on frozen section and fine needle aspiration. I found that the book covered most common entities in detail and I do not see how this area of pathology could have been covered any more extensively. Special mention must be made about the excellent illustrations used in this book. The text is supported by over 1000 high quality, colour illustrations. These include well chosen colour images of gross and microscopic pathology, in addition to numerous colour artwork illustrations that enhance the text. Pertinent ultrastructural images are also included.

This is not meant as a major criticism, but if there is any downside, it has to be the small size of the print, which was smaller than that of most other pathology textbooks.

This book is an absolute must for histopathologists reporting on specimens of the pancreas, gallbladder, extrahepatic biliary tract, and ampullary region. I also strongly recommend this book to pathology trainees and other clinicians. I predict that this book will become one of the standard pathology textbooks on pancreatic, gallbladder, and extrahepatic biliary diseases.

D Govender

\section{Worms and Human Disease - Second Edition}

Muller R. (£35.00.) Cabi Publishing, 2002. ISBN 0851995160.

This book is the second edition of Worms and disease: a manual of medical helminthology first published in 1975.

It covers this branch of parasitology in sufficient detail, especially because significant advances have been made since 1975 in immunology, molecular biology, diagnosis, and treatment aspects of this complex branch of medicine

The text is balanced by core information and additional descriptions without resorting to elaborate and bulky text, which would have increased costs and added little to its use as a ready reference.

It succeeds as "a practical guide in human helminthology for physicians and medical technologists", and will find favour among postgraduate students in tropical diseases, undergraduate medical, zoological, and tropical engineering students, and technologists.

However, it does not cover detailed pathology and aspects of differential diagnosis, perhaps reserved for more specialised textbooks, which may not appeal to the intended wider reader base.

The reader is given adequate references, most of which are derived from the 1980 to 2000 period.

The life cycles and illustrations (including maps and drawings) are useful, with commendable appendices at the end of the book (notably Appendix 2 dealing with the glossary of helminth terms and Appendix 3 which covers the location of helminths in the human body).

The overall impression is that the book is a worthy addition to any medical library, and it is highly recommended for students in both clinical and laboratory medicine, workers in 
these disciplines, and allied sciences where helminthology has a bearing.

A Essa

\section{Cancer Cytogenetics: Methods and Protocols}

Swansbury J, ed. (\$89.50.) Humana Press, 2003. ISBN 1588290808.

The discovery of the Philadelphia chromosome by Nowell and Hungerford in 1960 greatly stimulated interest in cancer cytogenetics. Once banding techniques were refined in the 1970s, the field of cancer cytogenetics blossomed and benefited tremendously from the wealth of information that was quickly amassed. Today, the field is still growing rapidly with the advent of molecular cytogenetic techniques, such as fluorescent in situ hybridisation (FISH), multicolour FISH, spectral karyotyping, and comparative genomic hybridisation. However, there are fewer cancer cytogenetics laboratories than clinical cytogenetics laboratories because of the lower demand for this service. This is changing rapidly, as new prognostic associations are constantly being discovered. Therefore, Dr John Swansbury aimed to help those wishing to start a cancer cytogenetics service by putting together Cancer cytogenetics: methods and protocols.

Dr Swansbury wrote most of the book himself, but excellent contributions were made by some very prominent cancer cytogeneticists. The book is designed such that a chapter of background material on a certain topic is followed immediately by a technical chapter on the same topic. Chapters included most of the main areas of interest in cancer cytogenetics, such as myeloid disorders, acute lymphoblastic leukaemia, other lymphoid disorders, solid tumours, and FISH. There is also a chapter on the interpretation of cytogenetic findings, which is extremely important in malignancies. The background chapters are generally well written in a simplistic way for the novice. Cancer cytogenetics can be a very intimidating field for those not familiar with it, and Dr Swansbury does a good job of introducing it. The technical chapters are quite comprehensive and also very well written, with step by step and easy to follow protocols. There are plenty of explanations and trouble shooting suggestions for the many things that can go wrong in a cancer cytogenetics service laboratory.

One of the drawbacks of the book is that it does not put enough emphasis on the importance of prognostic FISH markers in haematological disorders and solid tumours. This is a rapidly growing field, and FISH plays an important role not only in the diagnosis of a malignancy, but also in the prognosis and response to treatment. FISH plays such a large part in the cancer cytogenetics laboratory today that it would have been useful to spend more time on its clinical applications. The book could have benefited also from a chapter on quality control and quality assurance. The service laboratory is very different from a research laboratory, and one must be sure of the results that are reported. It would be best to implement quality control and quality assurance measures right from the start, rather than to change things after a mistake has been made. Quality measures are crucial in all aspects of the cancer cytogenetics service, from culture set up and harvesting, to metaphase analysis, FISH probe validation, right through to reporting.

This book is aimed at the novice and does a very good job in getting one started with a cancer cytogenetics service. However, nothing can replace experience, and it is highly recommended that anyone starting out in the field should visit an established laboratory to see first hand how things are set up. I have no hesitation in recommending this book to any cytogeneticist interested in expanding their service to include malignancies, or to anyone interested in starting up a cancer cytogenetics laboratory.

K Chun

\section{Manual of Clinical Microbiology, 8th Edition}

Murray P R, Baron E J, Jorgensen J H, et al, eds. (\$189.95.) ASM Press, 2003. 1555812554

The manual of clinical microbiology, published by ASM Press, is a favourite of mine because of its immense detail and vast coverage of the field. The first edition was published in 1970 , with subsequent editions following at four to six yearly intervals, and culminating in this 8th edition, which has been expanded into a two volume set with 141 chapters and 2113 pages, written by 230 authors and an international editorial board composed mainly of microbiologists from the USA.

The manual of clinical microbiology is a colossal resource, which is very well presented and beautifully illustrated. Volume includes sections on "General issues in clinical microbiology", "The clinical microbiology laboratory in infection detection, prevention and control", "Diagnostic technologies in clinical microbiology", "Bacteriology", and "Antibacterial agents and susceptibility test methods". Volume II includes sections on "Virology", "Antiviral agents and susceptibility test methods", "Mycology", "Antifungal agents and susceptibility test methods", "Parasitology", and "Antiparasitic agents and susceptibility test methods".

The chapter on "Mycobacterium: phenotypic and genotypic identification" is 24 pages long, contains 170 references, and begins with an extensive description of phenotypic identification tests for mycobacteria, with tabulated data for the various cultural and biochemical tests, along with 16 large colour photographs of macroscopic and microscopic colonial morphology. Then there is a short discussion of mycobacterial genomes, including reference to the propensity within the genome for the production of enzymes involved in fatty acid metabolism (as compared with Escherichia coli, for example), and the fact that the genus has an extremely clonal population structure, with genomic variation largely caused by insertion sequence movement rather than by point mutations. This leads into a section on "Genotypic identification of mycobacterial strains", which begins with an introduction describing the development of the polymerase chain reaction (PCR) and restriction endonuclease analysis for detection of mycobacteria and the seminal work of Amalio Telenti. This is followed by a discussion of aspects and uses of commercially available identification probes (AccuProbe and INNO-Lipa), genome sequencing, markers for species identification within the Mycobacterium tuberculosis complex, and direct amplification tests, including the amplified $M$ tuberculosis direct test and Amplicor PCR test. Following this are sections on "Strain typing", "Immunodiagnostic tests", "Quality assurance", and "Interpretation and reporting of results".

My only criticism is that occasional chapters are a little light. For example, the chapter on "Antifungal agents" is only 10 pages long and would have benefited from additional consideration of the relative merits of the recently expanded range of available antifungal agents.

In conclusion, I will continue to use this excellent and detailed resource in its updated form primarily as a reference text because of its comprehensive content, good organisation and therefore ease of access to relevant sections, beautiful presentation, and particularly its academic depth relating to the practice of clinical microbiology.

\section{J Kerr}

\section{Cytokines and Chemokines in Infectious Diseases Handbook}

Kotb M, Calandra T. (\$145.00.) Humana Press, 2003. ISBN 0896039080.

Cytokines are soluble protein molecules that facilitate communication between cells of the immune system, and as such, orchestrate immune responses required to eliminate or localise invading infectious agents. Therefore, these molecules have obvious relevance to the study of infectious disease.

This book is divided into sections on cytokines in infectious disease, Gram negative infection, Gram positive infection, mycobacterial infection, other bacterial infection, fungal infection, parasitic infection, viral infection, cytokines as therapeutic agents in infectious disease, and anticytokine based therapy in treatment of infectious disease.

Certain chapters contain comprehensive information that is well presented, such as that on cytokine patterns in severe invasive group A streptococcal infections. However, others are superficial and inadequate, such as that on cytokine gene polymorphisms and host susceptibility to infection. This chapter contains sections on tumour necrosis factor $\alpha$, interleukin l (IL-1), IL-lra and other cytokines. However, the possibilities for a chapter on this topic are extensive and should also include sections on at least interferon $\gamma$ (IFN $\gamma$ ) and IL-10.

The section on cytokines in viral infections is superficial, with chapters only on viroceptors, human immunodeficiency virus (HIV) infection, and viral hepatitis. Chapters one might expect in this section would be those on Epstein-Barr virus induced cytokines and the relevance of this to diseases such as cancer and rheumatoid arthritis; Kaposi's sarcoma associated herpesvirus and the relevance of its IL-6 homologue to lymphoma, etc, etc. It seems odd to have a section on cytokines in viral infection and then to consider only three examples.

The section on cytokines as therapeutic agents in infectious disease contains chapters on IFN $\gamma$, IL-2 for HIV, and the use of granulocyte colony stimulating factor/granulocyte-macrophage stimulating factor. The section on anticytokines as treatment considers only septic shock, streptococcal toxic shock, and necrotising fasciitis. 
Although the book could be very useful in some contexts, such as sepsis and HIV, it lacks overall depth and clarity of structure and remit.

J Kerr

\section{Differential Diagnosis by Laboratory Medicine}

Mesko D. ed. (£35.00): Springer-Verlag, 2003. ISBN 3540430571.

What do you do when you get phoned in your laboratory office by a clinical colleague asking you what are the 10 causes of a raised urine $\delta$ aminolevulic acid? Well, you could disconnect the phone and hope they don't call back, you could start gabbling and say you have never heard of it, or alternatively you could consult this book! This 1000 plus page text is, indeed, a treasure trove of useful laboratory facts.

The book covers thoroughly many laboratory parameters in various biological materials. Other useful features were a detailed description of medications and how these may interfere with laboratory tests, and a section listing laboratory findings in a variety of clinical conditions. I also found the tables of what sampling tubes were necessary for particular laboratory tests extremely helpful. To add to this there are tables of reference ranges for numerous laboratory tests and also conversion factors for changing conventional units to SI units.

This vademecum is written by a group of experienced laboratory workers and covers clearly many aspects of clinical biochemistry, haematology, microbiology, and immunology and is a worthy addition to any clinical laboratory's bookshelf. I heartily recommend

M Crook

\section{CALENDAR OF EVENTS}

\section{Surgical Pathology for the Practising} Pathologist

16-19 January 2004, Doubletree La Posada Resort, Scottsdale, Arizona, USA

Further details: Department of Continuing Education, Harvard Medical School, PO Box 825, Boston, MA 02117-0825, USA. (Tel: +1 617384 8600; Fax: +1 617384 8686; Email hms-cme@hms.harvard.edu)

\section{Surgical Pathology for the Practising} Pathologist: Selected Topics

26-29 March 2004, Sanibel Harbour Resort and Spa, Fort Myers, Florida, USA

Further details: Department of Continuing Education, Harvard Medical School, PO Box 825, Boston, MA 02117-0825, USA. (Tel: +1 617384 8600; Fax: +1 617384 8686; Email hms-cme@hms.harvard.edu)

\section{Medicare India}

6-8 April 2004, Pragati Maidan, New Delhi, India

Further details: Rob Grant, Kinex Log, 5 New Quebec Street, London W1H 7DD, UK (Tel: +44 (0) 207723 8020; Fax: +44 (0) 207 723 8060; Email: rob.grant@kinexlog.com; Website: www.medicare-expo.com or www. kinexlog.com)

\section{Diagnostic Histopathology of the Breast}

10-14 May 2004, Hammersmith Hospital (Imperial College Faculty of Medicine), London, UK

Further details: Wolfson Conference Centre, Hammersmith Hospital, Du Cane Road, London W12 0NN, UK. (Tel: +44 (0) 20 8383 3117/3227/3245; Fax: +44 (0) 208383 2428; Email:wcc@ic.ac.uk)

\section{Practical Pulmonary Pathology}

27-30 July, 2004, Brompton Hospital, London, UK

Further details: Professor B Corrin, Brompton Hospital, London SW3 6NP, UK. (Tel: +44 (0)20 7351 8420; Fax: +44 (0)20 7351 8293; Email: b.corrin@ic.ac.uk)

\section{ACP Management Course for Pathologists, 2004}

8-10 September 2004, Hardwick Hall Hotel, Sedgefield, County Durham, UK

Further details: V Wood, ACP Central Office, 189 Dyke Road, Hove, East Sussex BN3 1TL, UK. (Tel: +44 (0) 1273 775700; Fax: +44 (0) 1273 773303; Email: valerie@pathologists. org.uk

\section{CORRECTION}

The Scotland and Newcastle epidemiological study of Hodgkin's disease: impact on histopathological review and EBV status on incidence estimates. Jarrett RF, Krajewski AS, Angus B, et al. $J$ Clin Pathol 2003;56:811-6. In the key to fig $3 \mathrm{~A}$ EBV - should have been shown as triangles and $\mathrm{EBV}+$ as squares. 